\title{
Pinning-Controllability of Complex Networks
}

\author{
Francesco Sorrentino* ${ }^{*}$, Mario di Bernardo*, Franco Garofalo*, Guanrong Chen ${ }^{\dagger}$ \\ *University of Naples Federico II, Naples 80125, Italy \\ $\dagger$ City University of Hong Kong, P.R. China \\ ¥ Corresponding author. E-mail: fsorrent@unina.it
}

\begin{abstract}
We study the problem of controlling a general complex network towards an assigned synchronous evolution, by means of a pinning control strategy. We define the pinning-controllability of the network in terms of the spectral properties of an extended network topology. The roles of the control and coupling gains as well as of the number of pinned nodes are also discussed.
\end{abstract}

\section{INTRODUCTION}

The need of regulating the behavior of large ensembles of interacting units is a common feature of many physical, social and biological networks. For instance, many regulatory mechanisms have been uncovered in the context of biological, physiological and cellular processes, which are fundamental to guarantee the correct functioning of the whole network [1]. Examples include, in physiology, the synchronous beat of heart cells whose rhythm is generated by pacemaker cells situated at the sinoatrial node [2], the control of the respiratory rhythm played by synaptically coupled pacemaker neurons in the medulla [3] and the generation of rhythmic pacemaker currents by networks of interstitial cells of Cajal in the gastrointestinal tract of mammals [4]. In social networks, opinion dynamics are often driven by key-individuals termed as opinion leaders [5]. Other relevant examples, in biology, are the mechanisms through which cell cycles are controlled [6] and synchronized [7].

Understanding the fundamental nature of such regulatory mechanisms is therefore of utmost importance in Physics and Applied Science. Pinning control has been proposed in the literature [8, 9] as a fitting model to provide an insight into the regulatory mechanisms to control lattices and networks of coupled dynamical systems. The general idea behind pinning control is a selffeedback action (over a given reference evolution), acting on a limited subset of the dynamical systems placed at the network nodes. These nodes, also termed as reference sites or pinned sites, play the role of network leaders/pacemakers. Specifically, a direct control action is active only on these nodes and is propagated to the rest of the network through the coupling among the vertices.

In this paper, we define the new concept of pinningcontrollability (different from synchronizability) and derive a new quantity to assess this property for physical networks of interest. These findings have immediate theoretical and experimental relevance. By evaluating the pinning-controllability of a given network, one could decide the effectiveness of a pinning control strategy in terms of the strength of the required control action, the number of nodes to be pinned and the effects that a given topology of feedback connections can have on the entire network.

The rest of the paper is outlined as follows. In Sec.
II we introduce the pinning control formalism, apt to describing dynamical complex networks subject to a decentralized control action. In Sec. III a new methodology (based on the Master Stability Function approach [10]) is introduced to define the pinning-controllability of a given complex network of interest. Finally, a validation is presented in Sec. IV, where pinning control of scalefree complex networks of chaotic oscillators is studied through numerical simulations.

\section{PINNING CONTROL: AN OVERVIEW}

In recent years, synchronization of complex networks of coupled oscillators has been the subject of intensive research activity within the scientific community. A common assumption in such literature is that all the dynamical systems at the network nodes are identical, while the problem of synchronization of networks of non-identical oscillators has received much less attention. In particular, the Master Stability Function (MSF) approach, introduced in [10], has been successfully applied to synchronization of complex networks in a wide variety of situations (see e.g. [10, 11, 14, 15], to name a few contributions). Note that the MSF approach is valid under the hypothesis that all the vector fields at the network dynamical nodes are identical.

In this paper, we study networks in which two different layers of dynamical nodes coexist: the uncontrolled sites and the reference (controlled) ones. In particular, the latter plays the role of leading the whole network towards a given (desired) reference evolution.

Generally, we assume the controlled complex network to be described by the following set of equations:

$\frac{d x_{i}}{d t}=f\left(x_{i}\right)+\sigma \sum_{j=1}^{N} \mathcal{A}_{i j}\left(h\left(x_{j}\right)-h\left(x_{i}\right)\right)+\sigma \sum_{k=1}^{n} \delta\left(i-c_{k}\right) u_{i}$

$i=1, \ldots, N$, representing the behavior of $N$ identical dynamical systems coupled through the network edges.

The first term on the right side of (1) describes the state dynamics of the oscillator at each node, $\left\{x_{i}(t), i=\right.$ $1, \ldots, N\}$, via the nonlinear vector field $f\left(x_{i}\right)$; the second term represents the coupling among pairs of connected oscillators, through a generic output function 
$h\left(x_{i}\right)$, where the coupling gain $\sigma$ represents the overall strength of the interaction. Information about the weighed network topology is contained in the coupling matrix $\mathcal{A}$, whose entries $\mathcal{A}_{i j}$, are zero if node $i$ is not connected to node $j \neq i$, but are positive if there is a direct influence from node $i$ to node $j$, with $\left|\mathcal{A}_{i j}\right|$ giving a measure of the strength of the interaction. In what follows, we assume the matrix $\mathcal{A}$ to be irreducible (i.e. the associated digraph is globally connected). The last term on the right-hand side of (11) represents the pinning control action. This term is present only for $n=p N$ (usually $p \ll 1$ ) pinned nodes in the network, identified by the set $C=\left\{c_{1}, c_{2}, \ldots, c_{n}\right\}$. As commonly assumed in pinning control schemes, such nodes play the role of leading the others towards some desired reference evolution, say $s(t)$. Note that the control input $u_{i}=\varphi\left(x_{i}, s, t\right)$ has a direct influence only on the nodes belonging to the set of the reference sites $C$.

In what follows, we consider two different strategies for choosing the pinned nodes: (i) Random pinning: The $n$ pinned nodes are randomly selected with uniform probability from the set of all the network vertices. (ii) $S e$ lective pinning: The $n$ pinned nodes are firstly sorted according to a certain property at the network vertices (for instance, the vertex degree or betweenness centrality), then the nodes to be pinned are chosen in that particular order.

Hereafter, following [9], we choose the control input $u_{i}$ to be generated by a simple state-feedback law with respect to the reference evolution $s(t)$, which is assumed to satisfy $\frac{d s}{d t}=f(s)$. Thus, we set $u_{i}=\kappa_{i}\left(s-x_{i}\right)$ at every pinned node $c_{i}, i=1, \ldots, n$, where $\kappa_{i}$ is the control gain acting on node $c_{i}$.

Eq. (1) can be rewritten as

$$
\frac{d x_{i}}{d t}=f\left(x_{i}\right)-\sigma \sum_{j=1}^{N} \mathcal{L}_{i j} h\left(x_{j}\right)-\sigma \sum_{k=1}^{n} \delta\left(i-c_{k}\right) \kappa_{i}\left(x_{i}-s\right),
$$

$i=1, \ldots, N$, where the elements of the Laplacian matrix $\mathcal{L}$, are as follows: $\mathcal{L}_{i j}=-\mathcal{A}_{i j}$ if $i \neq j$ and $\mathcal{L}_{i i}=\sum_{j \neq i} \mathcal{A}_{i j}$ $\forall i$. Denote by $\left\{\lambda_{i}=\lambda_{i}^{r}+j \lambda_{i}^{i}\right\}$ the set of eigenvalues of $\mathcal{L}$ and assume they are ordered in such a way that $\lambda_{1}^{r} \leq \lambda_{2}^{r} \leq \cdots \leq \lambda_{N}^{r}$.

Note that in the specific case where $\mathcal{C}=\emptyset$, i.e. no reference sites are present in the network, it is possible to define the synchronization manifold, $\mathcal{S}:=\left\{x_{1}=x_{2}=\right.$ $\left.\ldots=x_{N}\right\}$, which is an invariant set for system (2) [10]. In this case, according to the choice of the coupling gain $\sigma$, all the oscillators in the network may settle over a solution belonging to $\mathcal{S}$ (not known a priori) and the network becomes synchronized. Interestingly, in [10, 11], the stability of the manifold $\mathcal{S}$ was related to $R^{N}=\frac{\lambda_{N}^{r}}{\lambda_{2}^{r}}$ and $M^{N}=\max _{j} \lambda_{j}^{i}$, suggesting an evaluation of the synchronizability of a generic network topology in terms of its spectrum. In particular, a network is said to be more (or less) synchronizable according to the width of the range of values of the coupling gain $\sigma$ for which synchronization is attained.

A different problem consists, instead, in driving a network of coupled dynamical systems towards a desired evolution determined a priori, by means of an appropriate control action. This is the case where $\mathcal{C} \neq \emptyset$, indicating the presence of some closed-loop equations within the set $\mathcal{C}$ in (2). Note that when $\mathcal{C} \neq \emptyset$, the evolution $x_{1}(t)=x_{2}(t)=\ldots=x_{N}(t)=s(t)$ becomes the only admissible solution in $\mathcal{S}$ for the controlled network described by (2). Thus, it is important to study the stability of such a solution, which corresponds in this context to the model reference chosen for the network.

It is worth noting here that the Master Stability Function approach cannot be applied directly to network (2), because of the presence of inhomogeneous dynamics at the controlled/uncontrolled nodes. In the next section we will provide an extension of the MSF approach to the case of dynamical networks subject to pinning control, described by the set of equations (2).

\section{EVALUATING THE PINNING-CONTROLLABILITY OF COMPLEX NETWORKS}

In this section, we propose for the first time that the approach presented in [10] can be extended to define and assess the pinning-controllability of a given network of interest. In particular, given a network described by (2), we define the network pinning-controllability in terms of the values of the coupling gain $\sigma$ and the control gains $\kappa_{i}, i=1, \ldots, n$, in (2), needed in order for the network to achieve the desired evolution $\frac{d s}{d t}=f(s)$. We shall seek to assess the pinning-controllability of network (2) by considering an extended network of $N+1$ dynamical systems $y_{i}$, where $y_{i}=x_{i}$ for $i=1,2, . ., N$ and $y_{N+1}=s$. In so doing, we assume that the desired common evolution $s$ is given by the state evolution of an extra virtual vertex, which is added to the original network. Then, we can rewrite (2) as

$$
\frac{d y_{i}}{d t}=f\left(y_{i}\right)-\sigma \sum_{j=1}^{N+1} \mathcal{M}_{i j} h\left(y_{j}\right), \quad i=1,2, \ldots, N+1,
$$

where $\mathcal{M}=\left\{\mathcal{M}_{i j}\right\}$ is an $(N+1)$-dimensional square matrix, with $\mathcal{M}_{i j}=$

$$
\left(\begin{array}{ccccc}
\mathcal{L}_{11}+\xi_{1} \kappa_{1} & \mathcal{L}_{12} & \cdots & \mathcal{L}_{1 N} & -\xi_{1} \kappa_{1} \\
\mathcal{L}_{21} & \mathcal{L}_{22}+\xi_{2} \kappa_{2} & \cdots & \mathcal{L}_{2 N} & -\xi_{2} \kappa_{2} \\
\vdots & & \ddots & & \vdots \\
\mathcal{L}_{N 1} & \mathcal{L}_{N 2} & \cdots & \mathcal{L}_{N N}+\xi_{N} \kappa_{N} & -\xi_{N} \kappa_{N} \\
0 & 0 & \cdots & 0 & 0
\end{array}\right)
$$

and $\xi_{i}=\sum_{k=1}^{n} \delta\left(i-c_{k}\right)$.

Note that $\mathcal{M}$ is an asymmetric zero row-sum matrix, with positive values along the main diagonal. At first, let us assume the matrix $\mathcal{A}$ to be symmetric, which ensures the matrix $\mathcal{M}$ to be diagonalizable [23]. Then, let $\left\{\mu_{i}=\right.$ 
$\left.\mu_{i}^{r}+j \mu_{i}^{i}\right\}$ be the eigenvalues of $\mathcal{M}$ and assume they are ordered in such a way that $\mu_{1}^{r} \leq \mu_{2}^{r} \leq \cdots \leq \mu_{N}^{r}$. By graph theory and linear algebra, we have $\mu_{i}^{r} \geq 0 \forall i$ [12]. Moreover, $\mu_{1}$ is the only null eigenvalue of $\mathcal{M}$.

Now, the arguments of the Master Stability Function approach [10] immediately apply to (3). In particular, by making use of the MSF, we can investigate the stability of the reference evolution, $y_{1}(t)=y_{2}(t)=\ldots=y_{N}(t)=$ $y_{N+1}(t)$, which can be evaluated in terms of the stability of $N+1$ independent blocks in the parameter $\alpha=\sigma \mu_{i}$, $i=1, \ldots, N+1$. Once the functional forms of $f$ and $h$ have been assigned, the MSF associates to each value of the complex parameter $\alpha=\alpha^{r}+j \alpha^{i}$, the maximum Lyapunov exponent of the system in each block. It can be shown that for a large class of systems (in terms of the dynamic function $f$ and the output function $h$ ), there exists a bounded zone of the complex plane centered on the real axis, for which the MSF is negative. Thus, the condition to be satisfied, in order to guarantee the stability of the desired common solution $s(t)$, is that all the $\sigma \mu_{i}, i=2, \ldots, N+1$, belong to the bounded region of the complex plane where the MSF is negative [10]. Namely, for a given form of the matrix $\mathcal{M}$, there is typically a finite range of values of $\sigma$, say $\Sigma$, for which the stability of the synchronous state can be achieved.

Hence, the spectrum of $\mathcal{M}$ affects the stability of the synchronization manifold. In this paper, we focus on the case that $\mathcal{M}$ has a real spectrum. Then, the lower the eigenratio $R^{N+1}=\frac{\mu_{N+1}^{r}}{\mu_{2}^{r}}$, the larger the $\Sigma[10]$. More generally, when the Laplacian matrix has a complex spectrum, it has been proposed that the width of $\Sigma$ can be evaluated in terms of both $R^{N+1}=\frac{\mu_{N+1}^{r}}{\mu_{2}^{r}}$ and $M^{N+1}=\max _{j} \mu_{j}^{i}[11]$. Namely, the condition on the maximum imaginary part of the spectrum gives information about the spread of the eigenvalues along the direction of the imaginary axis; in the limit of $M^{N+1} \rightarrow 0$, the entire spectrum tends to the real axis and the (best) condition is recovered that $\Sigma$ depends only on $R^{N+1}$. Furthermore, as explained in [13], the same approach can be extended also to the case of $\mathcal{M}$ being a nondiagonalizable matrix, when the condition is satisfied that the network embeds at least an oriented spanning tree [24].

Now, in the case often considered in the literature of all control gains being the same, i.e. $\kappa=\kappa_{1}=$ $\kappa_{2}=\ldots=\kappa_{n}$, by rewriting the system in (3) as $\frac{d y_{i}}{d t}=f\left(y_{i}\right)-\kappa \sum_{j=1}^{N+1} \mathcal{M}_{i j}^{\prime} h\left(y_{j}\right)$, for $i=1, \ldots, N+1$, where $\mathcal{M}^{\prime}=\frac{\sigma}{\kappa} \mathcal{M}$, and by noticing that the two matrices $\mathcal{M}$ and $\mathcal{M}^{\prime}$ are characterized by the same eigenratios, we obtain, for every choice of $\sigma$, a similar condition on the interval of values of $\kappa$, say $\mathcal{K}$, for which the reference evolution is stable (this is true as long as the maximum imaginary part $M^{N+1}$ remains negligible).

Note that, by taking this approach, we have succeeded in decoupling the dynamical properties of the open-loop network (in terms of $f$ and $h$ ) from the factors encoded in the matrix $\mathcal{M}$. Specifically: (i) the structural prop-
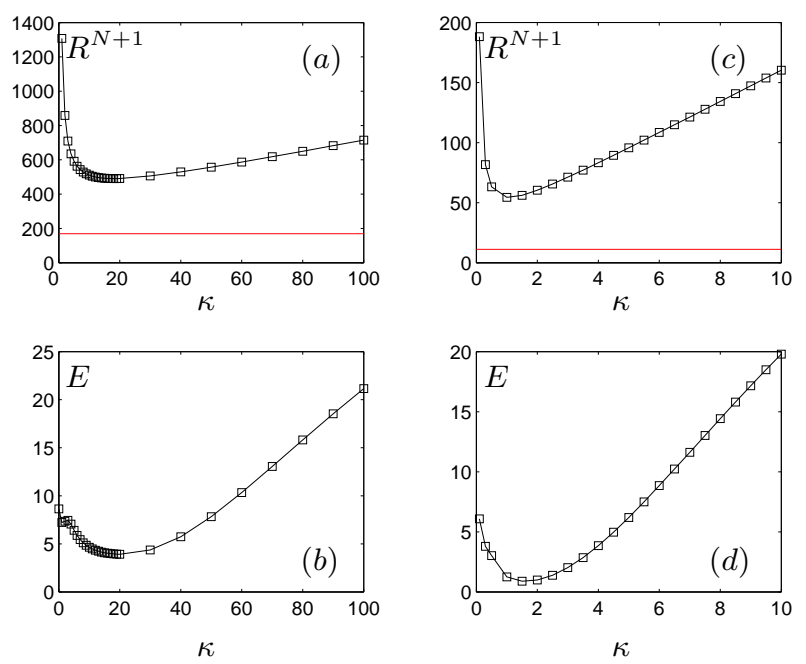

FIG. 1: Left pictures: Combinatorial Laplacian (symmetric topology). (a) Behavior of the eigenratio $\mu_{N+1} / \mu_{2}$ of the Laplacian spectrum as function of the control gain $\kappa$ for a BA network, with average degree $\langle k\rangle=2, p=0.1, \sigma=0.30$. (b) Control error at regime $E$ as function of the control gain $\kappa$ under the same conditions as in the upper plot.

Right pictures: Normalized Laplacian (asymmetric topology). (c) $R^{N+1}$ vs. $\kappa$ for a BA network, with average degree $\langle k\rangle=2$, $p=0.1, \sigma=2.8$. (d) $E$ vs. $\kappa$ under the same conditions as in the upper plot. In (a) and (c) the horizontal continuous lines represent the eigenratio $R^{N}$ of the corresponding uncontrolled networks.

erties of the network, in terms of the original network topology and the choice of the set of the controlled nodes $\mathcal{C}$, and (ii) the choice for each node in $\mathcal{C}, c_{1}, c_{2}, \ldots, c_{n}$ of the associated control gain $\kappa_{1}, \kappa_{2}, \ldots, \kappa_{n}$.

Thus, in analogy to the concept of network synchronizability defined in the literature [10, 14, 15], we propose to give a definition of the network pinning-controllability in terms of the widths of the ranges $\Sigma$ and $\mathcal{K}$, for which the reference evolution $y_{1}(t)=y_{2}(t)=\ldots=y_{N}(t)=s(t)$ is stable. Specifically, the lower the $R^{N+1}$ and $M^{N+1}$ are, the more the network is pinning-controllable (note that this definition is independent of the choice of the functions $f$ and $h$ ). Also, according to this definition, it becomes possible to act on both the network topology as well as the choice of the nodes to pin and their control gains, in order to vary (and eventually improve) the network pinning-controllability.

\section{NUMERICAL RESULTS}

In this section, by following the approach presented above, we present numerical evidence of the usefulness of $R^{N+1}$ as an index for evaluating the pinningcontrollability of a given complex network. Moreover, we show the behavior of $R^{N+1}$ under variations of the control gain $\kappa$ and the pinning probability $p$, in the case of scale-free complex networks. 

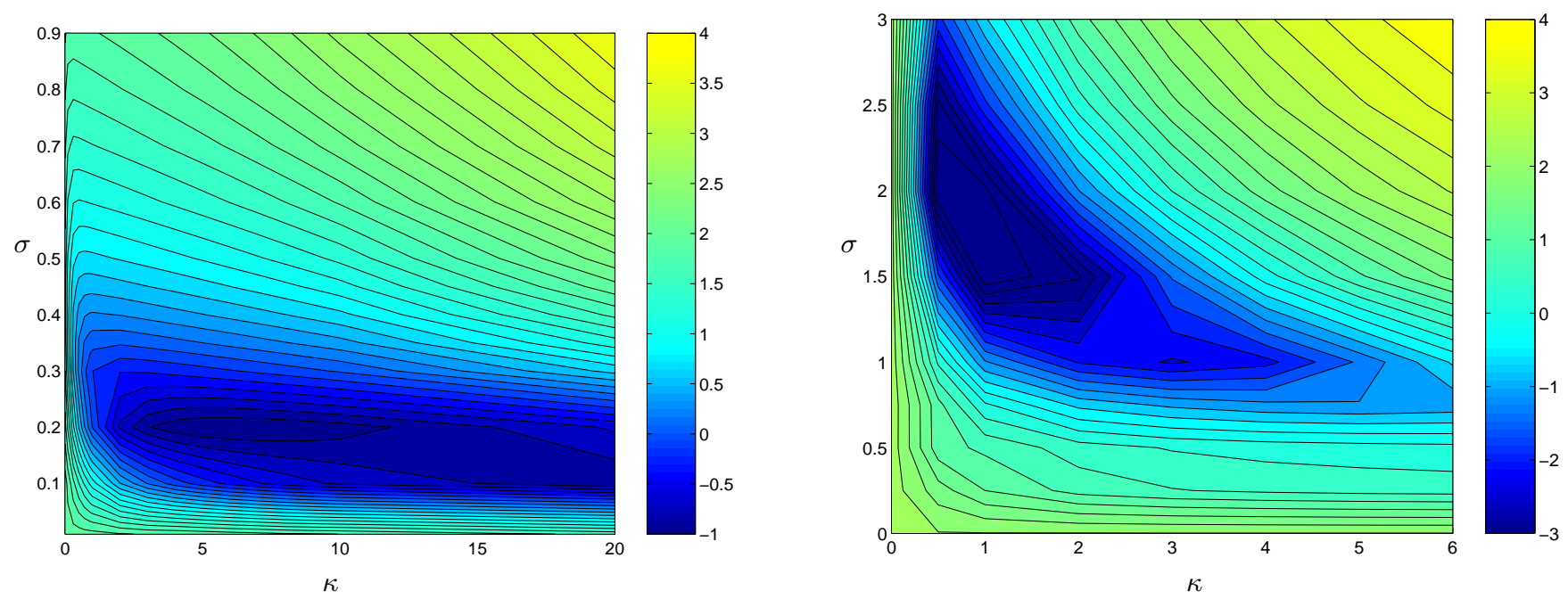

FIG. 2: (Color online) Curve level sets of the natural logarithm of $E$, asymptotic value of the control error, as a function of both $\kappa$ and $\sigma$, for a BA network of $10^{3}$ nodes, with average degree $\langle k\rangle=2, p=0.1$. The left (right) panel shows the case of Combinatorial (Normalized) Laplacian.

To validate our theoretical findings, we consider the case of a Barabasi-Albert (BA) scale-free network [16], characterized by a power-law degree distribution $P(k) \sim$ $k^{-3}$ (we have checked our results to be reproduced in a similar qualitative way for different kinds of networks and lattices, including real-world samples of networks), with $N$ identical Rössler oscillators placed at the network vertices. Namely, the dynamics at each node $i$ is described by the following vector field: $f\left(x_{i}\right)=$ $f\left(x_{i 1}, x_{i 2}, x_{i 3}\right)=\left(-x_{i 2}-x_{i 3}, \quad x_{i 1}+0.165 x_{i 2}, \quad 0.2+\right.$ $\left.\left(x_{i 1}-10\right) x_{i 3}\right)$. The output function $h$ has been chosen, as in [19], to be $h(x)=H x$, where $H$ is the matrix: $\left.\left(\begin{array}{lll}1 & 0 & 0\end{array}\right),\left(\begin{array}{lll}0 & 0 & 0\end{array}\right),\left(\begin{array}{lll}0 & 0 & 1\end{array}\right)\right)$, indicating that the oscillators are coupled through the variables $x_{i 1}$ and $x_{i 3}$ $(i=1,2, \ldots, N+1)$.

Two cases have been considered: (1) the Combinatorial Laplacian defined as $\mathcal{L}_{i j}=\mathcal{L}_{j i}=-1$ if $i$ and $j$ are connected $(i \neq j), 0$ otherwise, and $\mathcal{L}_{i i}=\kappa_{i} \quad \forall i$ (corresponding to a symmetric network); and (2) the Normalized Laplacian defined as $\mathcal{L}_{i j}=-1 / \kappa_{i}$ if $i$ is connected to $j(i \neq j), 0$ otherwise, and $\mathcal{L}_{i i}=1 \quad \forall i$, which corresponds to an asymmetric network configuration (for more details, see [12]).

For simplicity, we evaluate here the effect of $n$ pinned nodes with gains $\kappa_{1}=\kappa_{2}=\ldots=\kappa_{n}=\kappa$, on the structural parameters $R^{N+1}$ and $M^{N+1}$. For both the Combinatorial and the Normalized Laplacians considered here, the spectra are real and thus $M^{N+1}=0[25]$. The main results are shown in Fig. 1, for symmetric and asymmetric topologies. In both cases, the eigenratio $R^{N+1}$ (in Figs 1(a) and1(c)) is characterized by a minimum around a specific value of the control gain $\kappa$. This has immediate relevance as it suggests that by appropriately tuning the control gain, it is possible to enhance the pinningcontrollability of the network. Specifically, we observe that either a too large or too low value of $\kappa$ can reduce the network pinning-controllability.

As shown in Figs 1(a) and 1(c), when $p$ is low $(p=0.1$ in the figure), $R^{N+1}$ is higher than the eigenratio $R^{N}$ associated with the corresponding uncontrolled network (represented by the continuous horizontal lines in Fig (1). Intuitively this is not surprising since, in order to control the network, we are requiring one more eigenvalue (than in the case of generic network synchronization [10]) to fall into the stability region of the MSF.

Note that, for every choice of the coupling gain $\sigma$, the eigenratio $R^{N+1}$ may be conveniently varied by choosing an appropriate control gain $\kappa$. At the same time, for every $\kappa$, a bounded region of stability can be defined on the complex plane, and a timely choice of $\sigma$ is necessary in order to place all the $\sigma \mu_{i}$ inside it. Thus, the stability of the reference evolution is sensible to both the values of the control gain and the coupling gain and values of $\kappa$ and $\sigma$, which are either too large or too small, may prevent the stability over the reference evolution of the network trajectories.

It is worth noting here that there is a prominent difference between the effects that varying $\sigma$ and $\kappa$ can have on the pinning-controllability of a given network. Specifically the width of $\Sigma$ is determined essentially by the particular shape of the MSF (which can be numerically computed, once the forms of the functions $f$ and $h$ are given). On the other hand, the width of $\mathcal{K}$ depends on the spectral properties of the matrix associated to the extended network, defined as proposed in this paper. Specifically, when the MSF is non-monotone, as in the case of the $x z$ coupled Rössler discussed in this paper, the width of the interval of the values of $\kappa$, for which the stability of the reference evolution can be guaranteed, depends simply on the eigenratio $R^{N+1}$, as shown in Fig. 11(a)-(c).

The asymptotic value of the control error $E=$ $\frac{1}{(\Delta T) N} \sum_{i=1}^{N} \int_{T}^{T+\Delta T}\left\|x_{i}(t)-s(t)\right\| d t$, with $\|x\|=\left|x_{1}\right|+$ 

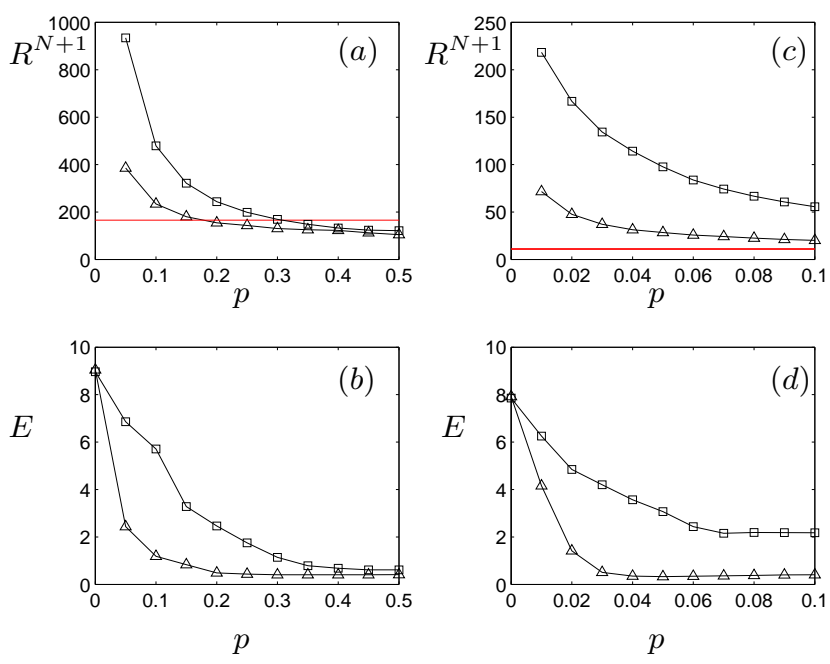

FIG. 3: Left pictures: Combinatorial Laplacian (symmetric topology). (a) Behavior of the eigenratio $\mu_{N+1} / \mu_{2}$ of the Laplacian spectrum as function of the control gain $\kappa$ for a BA network, with average degree $\langle k\rangle=2, \sigma=0.30, \kappa=10$. (b) Control error at regime $E$ as function of the control gain $\kappa$ under the same conditions as in the upper plot.

Right pictures: Normalized Laplacian (asymmetric topology). (c) $R^{N+1}$ vs. $\kappa$ for a BA network, with average degree $\langle k\rangle=2$, $\sigma=2.8, \kappa=1.5$. (d) $E$ vs. $\kappa$ under the same conditions as in the upper plot.

In all the plots the legend is as follows: squares (triangles) are used in the case of random (selective) pinning. In (a) and (c) the horizontal continuous lines represent the eigenratio $R^{N}$ of the corresponding uncontrolled networks.

$\left|x_{2}\right|+\left|x_{3}\right|$, has been computed under variations of the control gain $\kappa$ in Figs. 1(b) and 1(d) (respectively for fixed $\sigma=0.30$ and 2.8) and both $\kappa$ and $\sigma$ in Fig. 2. Note that interestingly the control error $E$ in Fig. 2 behaves locally as a convex function of $[\kappa, \sigma]$.

Finally, the effect of a variable number of pinned nodes (randomly selected within the network) is shown in Fig. 3. Observe that, by increasing the number of reference nodes, differently from the case of variable control gains, we recover a monotone behavior in the eigenratio $R^{N+1}$, as shown in Fig 3(a). Interestingly, by increasing $p$, it is even possible to make $R^{N+1}$ lower than $R^{N}$, i.e. controlling the network becomes easier than synchronizing it. Also in Fig. 3(b), the asymptotic value of the control error $E$ is observed to decrease for increasing values of $p$.

In Fig. 3 a comparison between random pinning (squares) and selective pinning (triangles) is also shown. In the case of selective pinning, the nodes have been chosen in the order of decreasing degree. We observe that selective pinning yields a lower eigenratio $R^{N+1}$ as well as a lower control error $E$ over different values of $p$, as shown separately in Figs. [3(a)-(b). This is in agreement with the previously reported results where a different approach was used to evaluate the effectiveness of pinning control schemes [9]. Moreover, this shows the benefits of taking into account some network topological features in choosing some suitable nodes to pin.

From a control design viewpoint, we observe that, while increasing the control gains may lead to a loss of pinning-controllability of the network, applying a larger number of controllers is always an effective strategy. On the other hand, the requirement of pinning a large number of nodes can make the technique unfeasible in those networks where altering the dynamics of too many nodes can lead to a loss of functionality of the network itself, or is too costly and unpractical.

The case of Normalized Laplacian is shown in Figs. 3(c)-(d), where the selective pinning is confirmed to perform better than the random one. This indicates that high degree vertices continue to be better suited to control the network than the others, even when the total strength of the interaction at each vertex is rescaled by its degree.

Following these preliminary results, we believe other network properties such as degree correlation [17], clustering [21], centrality [20] and community structure 22] can influence the effectiveness of pinning-control schemes. This represents the subject of future research activities.

\section{CONCLUSIONS}

In this paper we have presented a novel theoretical approach to describing the controllability of networks under pinning control schemes. We have defined the novel concept of pinning-controllability and characterized the pinning-controllability of a given network in terms of the coupling gain, the control gain and the number of pinned nodes. We found that this property can be analyzed by investigating the synchronizability of an appropriately extended network. For instance, Fig. 1 shows that there are values of the control gain which render the eigenratio or the control error minimal. We wish to emphasize that information such as this could be used to understand why some physical networks possess certain values of the coupling and control parameters. Moreover, the methodology presented here can be an effective tool for the design of pinning control schemes of many biological and technological networks.

Acknowledgments: This research was partially supported by the Hong Kong Research Grants Council under the CERG Grant CityU1114/05E.
[1] S. V. Vishnampet, Regulation and Control Mechanisms in Biological Systems (Prenctice Hall, 1992).
[2] C. Peskin, Mathematical aspects of heart physiology (Courant Inst. Math Sci., New York, 1977). M. Guevara 
and T. Lewis, Chaos 5, 174 (1995).

[3] N. Koshiya and J. C. Smith, Nature 400, 360 (1999).

[4] J. Thomsen, T. Robinson, J. Lee, L. Farraway, M. Hughes, D. Andrews, and J. Huizinga, Nature Medicine 4, 848 (1998).

[5] G. Weisbuch, G. Deffuant, and F. Amblard, Physica A 353, 555 (2004). Z. Neda, E. Ravasz, Y. Brechet, T. Vicsek, and A.-L. Barabasi, Nature 403, 849 (2000). M. McClintock, Nature 229, 244 (1971).

[6] L. H. Hartwell, J. Culotti, and B. Reid, PNAS 66, 352 (1970). R. Hunt, A. Hunter, and A. Munro, Nature 220, 481 (1968). P. Nurse, Nature 256, 547 (1975).

[7] R. A. Tobey, N. Oishi, and H. A. Crissman, PNAS 87, 5104 (1990). D. Battogtokh, K. Aihara, and J. Tyson, Phys. Rev. Lett. 96, 148102 (2006).

[8] R. Grigoriev, M. Cross, and H. Schuster, Phys. Rev. Lett. 79, 2795 (1997). N. Parekh, S. Parthasarathy, and S. Sinha, Phys. Rev. Lett. 81, 1401 (1998).

[9] X. Wang and G. Chen, Physica A 310, 521 (2002). X. Li, X. Wang, and G. Chen, IEEE Transa. on Circuits and Systems -I 51, 2074 (2004).

[10] L. Pecora and T. Carroll, Phys. Rev. Lett. 80, 2109 (1998).

[11] D. Hwang, M. Chavez, A. Amann, and S. Boccaletti, Phys. Rev. Lett. 94, 138701 (2005).

[12] F. Chung, Spectral Graph Theory (American Mathematical Society, 1997).

[13] T. Nishikawa and A. E. Motter, Phys. Rev. E. 73, 065106
(2006).

[14] T. Nishikawa, A. Motter, Y. Lai, and F. Hoppensteadt, Phys. Rev. Lett. 91, 014101 (2003).

[15] S. Boccaletti, V. Latora, Y. Moreno, M. Chavez, and D. Hwang, Physics Reports 424, 175 (2006).

[16] A. Barabasi and R. Albert, Science 286, 509 (1999).

[17] F. Sorrentino, M. di Bernardo, G. Huerta Cuellar, and S. Boccaletti Physica D 224, 123 (2006).

[18] A. Motter, C. Zhou, and J. Kurths, Europhys. Lett. 69, 334 (2005).

[19] M. di Bernardo, F. Garofalo, and F. Sorrentino, International Journal of Bifurcation and Chaos (in press).

[20] M.E.J. Newman, Phys. Rev. E 64, 016132 (2001).

[21] D.J. Watts, S.H. Strogatz, Nature 393, 440 (1998).

[22] M. Girvan, M.E.J. Newman, Proc. Natl. Acad. Sci. USA 99, 7821 (2002).

[23] In fact the spectrum of $\mathcal{M}$ can be decomposed in the spectrum of its minor containing its first $N$ rows and first $N$ columns, which is a symmetric matrix, plus one zero eigenvalue.

[24] The existence of an oriented spanning tree, having its root at the node $N+1$, is guaranteed by matrix $\mathcal{A}$ to be irreducible.

[25] Namely, in the case of Normalized Laplacian, this can be shown by checking that there exists a symmetric matrix similar to $\mathcal{M}$ [18]. 